\title{
Validação de uma tecnologia educacional: Manual obstétrico para a atenção primária
}

RESUMO | Objetivo: Elaborar e validar um manual obstétrico direcionado aos profissionais da atenção primária, do município de Marabá-PA. Método: Trata-se de pesquisa aplicada de tecnologia educacional, de natureza experimental que objetiva a criação de um manual a ser utilizado em consultas de pré-natal por profissionais da atenção primária. Após a confecção do manual seguiu-se com a validação. Neste trabalho foi utilizada a teoria da psicometria de acordo com Pasquali, optou-se por capturar 06 especialistas de acordo com a área de interesse para validação, sendo utilizada a escala tipo Likert. Resultados: Quanto à validação junto aos especialistas todos os itens foram considerados validados, haja vista terem apresentado IVC (Índice de Validade do Conteúdo) maior que 0,78 ou $78 \%$. Conclusão: Após o manual elaborado foi considerado validado em conteúdo e aparência por especialistas, sendo importante a realização de estudo posterior para verificar o seu impacto na cidade de Marabá na qual será utilizado.

Palavras-chaves: Tecnologia Educacional; Manual; Cuidado Pré-Natal.

ABSTRACT | Objective: Elaborate and validate an obstetric manual aimed at primary care professionals, from the municipality of Marabá-PA. Method This is applied research of educational technology, of experimental nature that aims to create a manual to be used in prenatal consultations by primary care professionals. After the manual was created, it was followed by validation. In this work the psychometry theory was used according to Pasquali, it was chosen to capture 06 specialists according to the area of interest for validation, using the Likert scale. Results: Regarding the validation with the experts, all items were considered validated, since they presented IVC (Content Validity Index) greater than 0.78 or $78 \%$. Conclusion: After the elaborated manual was considered validated in content and appearance by specialists, being important to carry out a later study to verify its impact in the city of Marabá where it will be used.

Keywords: Educational Technology; Guide; Prenatal Care.

RESUMEN | Objetivo: Elaborar y validar un manual obstétrico dirigido a los profesionales de la atención primaria, del municipio de Marabá-PA. Método Se trata de investigación aplicada de tecnología educativa, de naturaleza experimental que objetiva la creación de un manual a ser utilizado en consultas de prenatal por profesionales de la atención primaria. Después de la confección del manual se siguió con la validación. En este trabajo se utilizó la teoría de la psicometría de acuerdo con Pasquali, se optó por capturar a 06 especialistas de acuerdo con el área de interés para validación, siendo utilizada la escala tipo Likert. Resultados: En cuanto a la validación junto a los especialistas, todos los ítems se consideraron validados, habiéndose visto que el índice de validez del contenido (índice de validez del contenido) mayor que 0,78 o 78\%. Conclusión: Después del manual elaborado fue considerado validado en contenido y apariencia por especialistas, siendo importante la realización de estudio posterior para verificar su impacto en la ciudad de Marabá en la que será utilizado.

Descriptores: Tecnología Educativa; Manual; Cuidado Prenatal.

\section{Kecyani Lima dos Reis}

Enfermeira assistencial. Docente Faculdade Carajás. Mestra pelo Mestrado Profissional em Cirurgia e Pesquisa Experimental - CIPE. Universidade Estadual do Pará - UEPA

\section{Percília Augusta Santana da Silva}

Enfermeira assistencial. Docente Faculdade Carajás, Mestra pelo Mestrado Profissional em Cirurgia e Pesquisa Experimental - CIPE .Universidade Estadual do Pará - UEPA

\section{Mariseth Carvalho de Andrade}

Estaticista, Mestra pelo Mestrado Profissional em Cirurgia e Pesquisa Experimental - CIPE. Universidade Estadual do Pará - UEPA

\section{Anderson Bentes de Lima}

Farmacêutico.Doutor .Professor Assistente da Universidade do Estado do Pará- UEPA.

\section{Marcus Vinicius Henriques Brito}

Médico.Doutor. Professor Titular da Universidade do Estado do Pará - UEPA. Coordenador da Capes.

\section{Nara Macedo Botelho}

Médica. Doutora. Professora Titular da Universidade do Estado do Pará - UEPA.

Recebido em: 29/04/2019

Aprovado em: 26/05/2019

\section{INTRODUÇÃO}

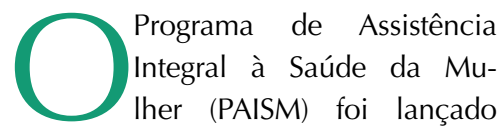
em 1983, o qual viabilizou o acesso da população a medidas de contracepção, integrando ainda a assistência às ações educativas, preventivas, diagnósticas e de tratamento, tanto no que concerne à especialidade da ginecologia como também no âmbito do pré-natal, parto, puerpério, climatério, planejamento familiar, Doenças Sexualmente Transmissíveis (DSTs) e cânceres de mama e colo-uterino ${ }^{(1)}$.

Em março de 2011, foi lançado pelo Ministério da Saúde o programa nacional intitulado Rede Cegonha, que tem por 
objetivo prestar atendimento adequado, seguro e humanizado desde a confirmação da gravidez, passando pelo pré-natal, parto e puerpério, até os dois primeiros anos de vida da criança ${ }^{(2)}$.

No município de Marabá, o programa da Rede Cegonha foi implantado em junho de 2012 e, em 2014, a atenção primária realizou o treinamento do sistema do pré-natal (SISPRENATAL), sendo que este, ainda se encontra em processo de instalação gradativa. No momento, todas as unidades básicas estão efetivando o programa com a atuação dos enfermeiros e dos médicos na consulta de assistência ao pré-natal.

O pré-natal é caracterizado pelo acompanhamento da mulher grávida, desde o início da gestação que em 90\% das mulheres é diagnosticado pela ausência de menstruação e sintomas clássicos como enjoos e vômitos matinais, visando manter a integridade das condições de saúde da mãe e do bebê. Assim, durante toda a gravidez serão realizados exames e avaliações complementares com vistas a identificar e tratar precocemente as situações de risco que podem trazer prejuízos à saúde da mãe ou da criança ${ }^{(4,5)}$.

O acompanhamento do pré-natal na rede básica de saúde é realizado pelo enfermeiro e pelo médico, com objetivo de monitorar, prevenir e identificar intercorrências maternas e fetais e, ainda, realizar atividades educativas acerca da gravidez, parto e puerpério. No entanto, compete ao enfermeiro o acompanhamento das mulheres com ausência de complicações, cadastradas no pré-natal de baixo risco ${ }^{(5)}$.

De acordo com o Sistema de Informação da Vigilância Epidemiológica do Município de Marabá, a porcentagem de óbitos por causas perinatais em relação ao número de nascidos vivos no período de 2012 a 2016 configurou-se da seguinte forma: 2012 - 62,17\%, 2013 - 140,84\%, 2014 - 77,83\%; 2015 - 38,61\% e 2016 $42,12 \%$. No ano de 2016, foi obtido um total de 4229 (100\%) de gestantes com a ficha de pré-natal preenchida, mas somente $17,09 \%$ com 1 a 3 consultas, 43,48\%

com 4 a 6 consultas e 39,41\% com mais de 7 consultas durante a gestação.

É necessário planejar a organização da rede regional de atenção à saúde para garantir o acesso e o acolhimento de todas as mulheres durante as diversas fases do ciclo gravídico puerperal, desenvolvendo atividades de promoção à saúde e de prevenção, cura e reabilitação dos agravos e patologias eventualmente apresentados nesse período, incluindo os cuidados com o recém-nascido. Para isso, é preciso haver integração entre as diversas unidades de atenção à saúde, garantindo retaguarda ambulatorial especializada e hospitalar, se for o caso $^{(8)}$.
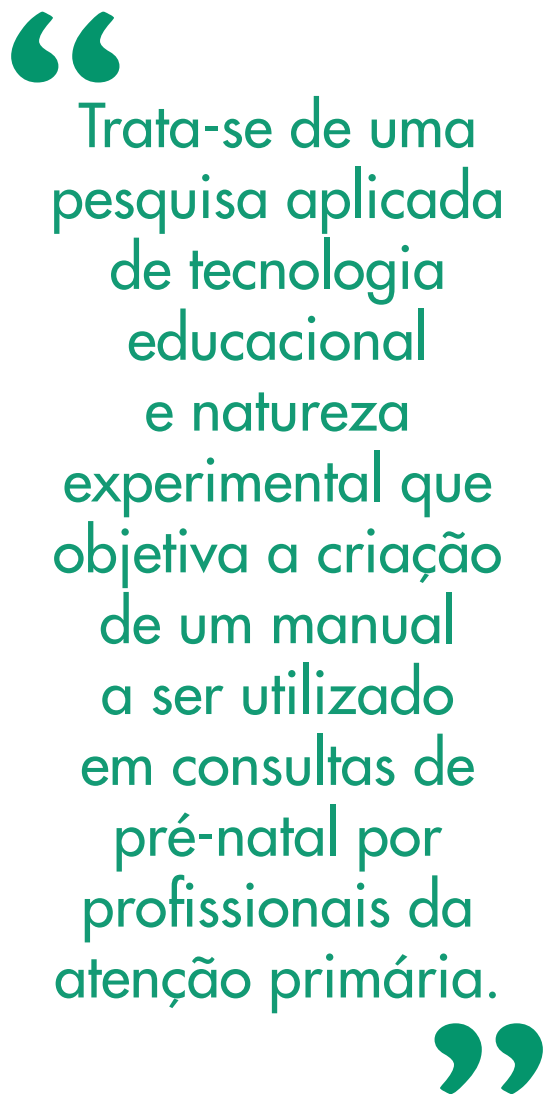

A busca pela qualidade da assistência passa pela organização e o gerenciamento dos serviços. Nesta perspectiva, a criação de manuais direcionados a profissionais da área da saúde pode ser considerada uma ferramenta facilitadora no processo de cuidar, pois à medida que as condutas assistenciais tornam-se atua- lizadas e padronizadas, a qualidade do atendimento tende a crescer. Logo, a elaboração de um manual que vise orientar as condutas dos profissionais na atenção primária para a assistência pré-natal poderá contribuir para o aumento da adesão das gestantes aos serviços de saúde, aumentando a cobertura pré-natal, dessa forma, garantindo maior qualidade no acompanhamento e, consequentemente, contribuindo para a redução da mortalidade materna ${ }^{(9)}$.

Neste sentido, o problema de nossa pesquisa é: Como podemos elaborar e validar um manual que vise orientar as condutas dos profissionais na atenção primária para a assistência do pré-natal? Nosso objetivo é elaborar e validar um manual obstétrico sobre o pré-natal para os profissionais da atenção primária, do município de Marabá-Pará.

\section{METODOLOGIA}

Trata-se de uma pesquisa aplicada de tecnologia educacional e natureza experimental que objetiva a criação de um manual a ser utilizado em consultas de pré-natal por profissionais da atenção primária. Esta pesquisa teve caráter prospectivo e a forma de abordagem foi quantitativa analítica, envolvendo sujeitos e local.

Os aspectos éticos foram respeitados em todas as etapas do estudo, em concordância com a Convenção de Helsinki, e de acordo com as Resoluções n. ${ }^{\circ}$ 466/12 e 510/16 que tratam das normas para pesquisas envolvendo seres humanos. $\mathrm{O}$ presente trabalho foi submetido à apreciação pelo Comitê de Ética em Pesquisa (CEP), através da Plataforma Brasil, por se tratar de pesquisa envolvendo seres humanos, do Centro de Ciências Biológicas e da Saúde da Universidade do Estado do Pará (UEPA) com o parecer de aprovado em 30 de novembro de 2017 sob o n. ${ }^{\circ}$ 2.406.696, de forma que, após a sua aprovação, foi iniciada a coleta de dados em fevereiro de 2018, conforme prevê a resolução (CAAE: 79972117.5.0000.5174).

Os participantes do trabalho foram 
06 profissionais da área de saúde, especialistas em ginecologia e obstetrícia, saúde da mulher, que atuam na atenção básica do município de Marabá.

O manual será utilizado por profissionais que atuam nas Unidades Básicas de Saúde (UBS) do município de Marabá-PA. Esta cidade faz parte da Mesorregião Sudeste do Estado do Pará, e apresenta 23 UBS com 12 localizadas na zona urbana e 11 na zona rural. As Unidades Básicas do município de Marabá são compostas por equipes de Estratégia de Saúde da Família (ESF) e Estratégias de Agentes Comunitários de Saúde (EACS), que atuam de acordo com os programas do Ministério da Saúde (hipertensão e diabetes, tabagismo, tuberculose, hanseníase, saúde da mulher, saúde da criança, saúde da família, imunizações, saúde do idoso e saúde na escola).

O manual foi elaborado de acordo com as diretrizes e manuais do Ministério da Saúde, documentos do Conselho Federal de Enfermagem (COFEN), ConseIhos Regionais de Enfermagem (COREN), Federação Brasileira das Sociedades de Ginecologia e Obstetrícia (FEBRASGO) e artigos científicos, priorizando as revisões sistemáticas com metanálise e os ensaios clínicos randomizados, por serem esses estudos de maior nível de evidência pelo município de Marabá. Devido respeitar normas e rotinas do Sistema Único de Saúde (SUS), por se tratar de atenção primária de saúde relacionada à saúde da mulher.

A estrutura do manual foi de acordo com a necessidade de capacitar os profissionais e de fornecer o referenciamento e fluxos dentro do município. O manual é composto de onze capítulos que foram desenvolvidos para responder todas as indagações dos profissionais durante a consulta do pré-natal na rede de atenção primária.

Após a confecção do manual, foi realizada a segunda etapa da pesquisa, a validação. Este processo propôs como objetivo a avaliação da qualidade do material construído e de instrumentos. É recomendado que seja realizada a validação por profissionais da área de saúde, especialistas em ginecologia e obstetrícia e/ou saúde da mulher, como enfermeiros ou médicos especialistas nesta área. Neste estudo, optou-se pela validação junto a profissionais da saúde, com titulação mínima de especialista e pelo menos dois anos de experiência na área específica do material didático elaborado, e tendo como base critérios elaborados por Fehring ${ }^{(10)}$ e adaptados para este estudo.

Quanto ao número ideal de especialistas para o processo de validação de conteúdo, a literatura é diversificada. Os trabalhos de Pasquali ${ }^{(11)}$ e Bertoncello ${ }^{(12)}$ ressaltam que o número de seis especialistas é o recomendável para a validação do processo. Neste trabalho optou-se por capturar 06 especialistas para validar o manual do pré-natal.

Os profissionais foram identificados por meio do currículo lattes, considerando os critérios de inclusão: com titulação de enfermeiro ou médico especialista na área de ginecologia e obstetrícia, que possua vínculo com o município, ter tempo de serviço de no mínimo dois anos e local de atuação na rede municipal na atenção primária e/ou secundária.

Para a validação da primeira fase, foi utilizada a escala tipo Likert, que tem como objetivo alcançar o consenso entre um grupo de profissionais sobre um tema específico. O funcionamento da técnica de se dá por diversas aplicações de questionários baseados em Likert, aplicado em um grupo de especialistas na área de estudo, que devem permanecer em anonimato. Um feedback com as respostas do grupo e aprimoramento do instrumento é realizado, visando obter o consenso de todos os profissionais especialistas. Foi solicitado aos seis profissionais para lerem e sugerirem modificações quanto ao conteúdo, clareza (coerência) e linguagem de cada um dos tópicos do manual. $\mathrm{O}$ instrumento que foi utilizado caracteriza-se por uma escala com quatro pontos, sendo 1 (totalmente adequado), 2 (adequado), 3 (parcialmente adequado) e 4 (inadequado). Caso o profissional opte pelos pontos 3 ou 4, este terá que sugerir as modificações necessárias para adequação do manual ${ }^{(13)}$.

Posteriormente, foram contatados e explicados os objetivos do estudo. Após a concordância, a pesquisadora principal aplicou o Termo de Consentimento Livre e Esclarecido (TCLE) em duas vias de igual teor, sendo uma cópia para o pesquisador e outra para o especialista e entregou o instrumento de avaliação, bem como o manual sobre o pré-natal para o profissional, sendo solicitado o retorno em até 15 dias.

Para a validação do manual, foi utilizado o Índice de Validade do Conteúdo (IVC) tendo que ser igual ou superior a 0,78 ou $78 \%$. O IVC mede a concordância entre as opiniões dos especialistas ${ }^{(14)}$. Esse método emprega a escala do tipo Likert com pontuação de um a quatro. $\mathrm{O}$ escore do índice é calculado por meio da soma de concordância dos itens que foram marcados como "1 e 2 " pelos especialistas, dividido pelo número total de respostas. Para avaliar o manual de forma geral foi utilizada uma das formas de cálculo recomendada por Polit e Beck ${ }^{(14)}$, na qual o somatório de todos os IVC calculados separadamente é dividido pelo número de itens do instrumento, devendo o valor ser também superior a 0,78ou $78 \%$ para ser considerado validado.

As informações da caracterização amostral foram apuradas em um banco de dados elaborado no software Microsoft Office Excel ${ }^{\circledR} 2010$.

Na aplicação da Estatística Descritiva, foram construídos tabelas e gráficos para apresentação dos resultados e calculadas as medidas de posição como média aritmética e desvio padrão.

\section{RESULTADOS E DISCUSSÃO}

De acordo com o manual de atenção ao pré-natal de baixo risco ${ }^{(2)}$, os Estados e municípios necessitam dispor de uma rede de serviços organizada para a atenção obstétrica e neonatal, com mecanismos estabelecidos de referência e contrarreferência (retorno da gestante para atenção primária). 
A utilização do manual vem para facilitar a orientação da equipe multiprofissional, bem como uniformizar a informação por meio de uma linguagem fácil para um melhor entendimento do paciente e com eficácia comprovada(15).

O protocolo é um instrumento normativo que orienta os profissionais na realização de suas funções. Ele tem como base conhecimentos científicos e práticos do cotidiano do trabalho em saúde, de acordo com uma realidade extremamente dinâmica, o que o obriga, necessariamente, a ser avaliado permanentemente e modificado segundo as circunstâncias envolvidas. Todo ano o Ministério da Saúde lança manual e cadernetas com intuito de orientar essas consultas ao pré-natal(16).

$\mathrm{Na}$ primeira etapa de validação, os seis profissionais tinham idade entre 30 e 45 anos, com uma média de 35,8 anos. Todos os profissionais eram do sexo feminino (100\%). Em relação à formação, todos tinham especialização, sendo 03 em $\operatorname{ESF}(50 \%)$ e 03 em Ginecologia e Obstetrícia (50\%). Uma tinha mestrado e 02 estavam cursando o mestrado. Quanto aos anos de atuação, as enfermeiras trabalhavam entre 12 e 22 anos, com uma média de 15,6 anos e as médicas trabalhavam entre 11 a 25 anos, com uma média de 14,6 anos. Todos dos profissionais trabalhavam em Unidade de Atenção Primária de Saúde, especificamente na área de saúde da mulher. Quanto à validação do conteúdo do manual informativo, utilizando-se a escala tipo Likert para se obter o consenso en- tre os especialistas de acordo com cada item do questionário.

Observa-se que os especialistas reúnem em si experiência prática aliada a cursos de pós-graduação e/ou atuação na docência, fato que contribui para um processo de validação mais criterioso de modo que se verifica a capacidade que possuem para julgarem a adequabilidade do manual.

Inicialmente, os especialistas fizeram uma avaliação dos objetivos do manual, o qual se refere aos propósitos, metas ou fins que desejam atingir com a utilização do material. Quanto aos objetivos, todos os itens foram considerados validados, haja vista terem apresentado IVC maior que 0,78 ou $78 \%$. Ressalta-se que a maioria dos especialistas classificou os itens como totalmente adequados.

Tabela 1. Objetivos. Marabá, PA, Brasil, 2018

Avaliação do Manual: 1 - OBJETIVOS

Parcialmente Adequado Totalmente Adequado

1.1 - São coerentes com as necessidades dos profissionais: médico e enfermeiro na atenção primária durante as consultas de pré-natal.

1.2 - São coerentes do ponto de vista da assistência no pré-natal.

1.3 - Pode circular no meio científico na área da obstetrícia.

$16.7 \%$

1.4 - Atende aos objetivos da atenção primária que trabalha com assistência no pré-natal.

$--\cdot-$

$83.3 \%$

Após análise e avaliação dos objetivos, foi dado seguimento com a avaliação da estrutura e apresentação do manual, as quais se referem à forma de apresentar as orientações, incluindo sua organização geral, estrutura, estratégia de apresentação, coerência e formatação. No quesito estrutura e apresentação, o manual também foi considerado validado em todos os itens, pois o menor valor para o IVC foi de 0.83 ou $83 \%$ para o subitem 2.9 relacionado às figuras. Mais uma vez, mais da metade dos avaliadores classificou os itens como totalmente adequados, fato que colabora para o processo de validação desse material.

\section{Tabela 2. Estrutura e Apresentação. Marabá, PA, Brasil, 2018}

Avaliação do Manual: 2 - ESTRUTURA e APRESENTAÇÃO Inadequado Parcialmente Adequado Totalmente Adequado

2.1 - 0 manual serve de suporte de orientação aos enfermeiros e médicos durante a realização das consultas de pré-natal.

2.2 - As mensagens estão apresentadas de maneira clara e objetiva.

2.3 - As informações apresentadas estão cientificamente corretas.

2.4 - Apresenta sequência lógica do conteúdo proposto.

- 1 - $100 \%$

2.5 - As informações estão bem estruturadas em concordância e ortografia.

2.6 - As informações da capa, contracapa e apresentação são coerentes.

2.7 - 0 tamanho do título e dos tópicos está adequado.

2.8 - 0 número de páginas está adequado.

2.9 - As figuras são expressivas e suficientes.

\begin{tabular}{l}
---- \\
\hline---- \\
---- \\
---- \\
---- \\
---- \\
----
\end{tabular}

$----$

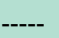

$100 \%$

$100 \%$

$100 \%$

$100 \%$

$100 \%$

$100 \%$

$100 \%$

$100 \%$

$16.7 \% \quad 83.3 \%$ 
A última etapa do processo de validação do manual foi referente à relevância do material, a qual avalia o grau de significação do material apresentado. O manual foi considerado validado no tocante à relevância, com variação do IVC de 1,0 ou 100\%.

Tabela 3. Relevância. Marabá, PA, Brasil, 2018

Avaliação do Manual: 3 - RELEVÂNCIA

Totalmente Adequado

3.1 - Os temas retratam aspectos-chave que devem ser reforçados.

$100 \%$

3.2 - 0 manual propõe aos profissionais da atenção primária adquirir mais conhecimento quanto às condutas adequadas durante as consultas de pré-natal.

$100 \%$

3.3 - 0 manual aborda assuntos necessários a serem oferecidos às gestantes em acompanhamento pré-natal.

$100 \%$

3.4 - Está adequado para ser usado por enfermeiros e médicos durante as consultas de pré-natal.

$100 \%$

Ao final do instrumento de avaliação, que foi entregue aos especialistas, foi destinado espaço para que fossem feitas sugestões visando o aperfeiçoamento do material. Foi sugerido também que, caso necessário, escrevessem observações no próprio manual. A partir dessas considerações, foram realizadas as modificações pertinentes. Para se contar com um instrumento validado com o rigor científico, que possa ser usado com plena confiança para futuras investi- gações, é preciso haver uma série de provas denominadas psicométricas. Algumas delas estão relacionadas à validade, comumente definida como a capacidade da prova de medir o que se intenta medir ${ }^{(17)}$.

A participação de especialistas no assunto e indivíduos que receberão as informações aumenta a credibilidade e melhora o conteúdo do manual obstétrico, tornando-o com uma linguagem acessível ${ }^{(18)}$.

\section{CONCLUSÃO}

O manual foi elaborado e considerado válido pelos profissionais especialistas que atuam na Atenção Primária de Marabá-PA. A elaboração de tecnologias educativas que visem desenvolver e melhorar o conhecimento técnico-científico de profissionais da saúde na assistência deve ser incentivada, pois promove a qualificação profissional, fortalecendo a prática assistencial da equipe da unidade básica de saúde no atendimento da gestante.

\section{Referências}

1. Freitas LV, Teles LMR, Lima TM, Vieira NFC, Barbosa RCM, Pinheiro AKB, et al. Physical examination during prenatal care: construction and validation of educational hypermedia for nursing. Acta Paul Enferm. 2012; 25(4):581-8. 2. Ministério da Saúde (BR). Portal da Saúde. Rede Cegonha [Internet]. Diretrizes Gerais e Operacionais da Rede Cegonha. Brasília, 2013 [acesso em 18 abr 2017]. Disponível em: http://portal.saude.gov.br/portal/saude/gestor/ visualizar_texto.cfm?idtxt=37082.

3. Ministério da Saúde, Secretaria de Atenção à Saúde, Departamento de Atenção Básica (BR). Atenção ao pré-natal de baixo risco / Ministério da Saúde. Secretaria de Atenção à Saúde. Departamento de Atenção Básica. Brasília: Ministério da Saúde; 2012.

4. Vieira, S. M. Percepção das puérperas sobre a assistência prestada pela equipe de saúde no pré-natal. Texto contexto - enferm. 2011; 20(spe).

5. Dotto LMG, Moulin NM, Mamede MV. Assistência pré-natal: dificuldades vivenciadas pelas enfermeiras. Rev. Latino-Am. Enfermagem. 2006 out.; $14(5)$.

6.Ministério da Saúde (BR). Protocolos da Atenção Básica: Saúde das MuIheres / Ministério da Saúde, Instituto Sírio-Libanês de Ensino e Pesquisa. Brasília: Ministério da Saúde; 2016.

7.Organização Pan-Americana da Saúde. Indicadores básicos de saúde no Brasil: conceitos e aplicações/Rede Interagencial de Informações para a Saúde - Ripsa. Brasília, Publicação da OPAS; 2002.

8.Ministério da Saúde, Secretaria de Atenção à Saúde, Departamento de Ações Programáticas Estratégicas (BR). Gestação de alto risco: manual técnico. 5. ed. Brasília: Ministério da Saúde; 2010.

9. Moraes,J.R. Protocolo para a consulta de enfermagem no pré-natal: cons- trução e validação. Dissertação (Mestrado) - Faculdade de Farmácia, Odontologia e Enfermagem, Universidade Federal do Ceará, Fortaleza, 2013.

10. Fehring, RJ. The Fehring model. In: Carool-Jonhnson RM, Paquete M, editor. Classification of nursing diagnoses: Tenth Conference. Philadelphia: JB Linppincott; 1994

11. Pasquali L. Psicometria:teorias e aplicações. Brasília: Unb; 1998.

12. Bertoncello KCG. Qualidade de vida e a satisfação da comunicação do paciente após laringectomia total: construção e validação de um instrumento de medida. 2004. $247 \mathrm{f}$. Tese (Doutorado em Enfermagem Fundamental) - Escola de Enfermagem de Ribeirão Preto, Universidade de São Paulo, Ribeirão Preto, 2004.

13. Maciel BS, Barros ALBL, Lopes JL. Elaboração e validação de um manual informativo sobre cateterismo cardíaco. Acta Paul Enferm. 2016; 29(6):633-42.

14. Polit DF, Beck CT, Hungler BP. Avaliação da Mensuração e da qualidade dos dados. In: __ . Fundamentos de pesquisa em Enfermagem: métodos, avaliação e utilização. 5. ed. Porto Alegre: Artmed; 2004. p. 285-306. 15. Lopes JL, Barbosa DA, Nogueira-Martins LA, Barros AL. Nursing guidance on bed baths to reduce anxiety. Rev Bras Enferm. 2015; 68(3):437-43.

16. Garces, S. B. B. Classificação e Tipos de Pesquisas. Cruz Alta: Unicruz; 2010.

17. Rodriguez MA, Lopera J. Conceptos básicos de La validación de escalas em salud mental. CES Medicina. 2002; 16(3):31-39.

18. Teles LMR, et al. Construção e validação de manual educativo para acompanhantes durante o trabalho de parto e parto. Rev Esc Enferm USP. 2014; 48(6):977-84. 